\title{
NONEXISTENCE OF NONTRIVIAL $\square$ "-HARMONIC 1-FORMS ON A COMPLETE FOLIATED RIEMANNIAN MANIFOLD
}

\author{
BY
}

HARUO KITAHARA

\begin{abstract}
We study the nonexistence of nontrivial $\square$ "-harmonic 1 -forms on a complete foliated riemannian manifold with positive definite Ricci curvature. It is well known that the harmonic 1-form on a compact and orientable riemannian manifold with positive definite Ricci curvature is trivial. Our main theorem is an extension of this fact in the complete foliated riemannian case.
\end{abstract}

Introduction. B. L. Reinhart [4] showed that on a compact foliated manifold $M$ with " bundle-like" metric, the cohomology of basic differential forms is isomorphic to the harmonic space of a certain semidefinite Laplacian. I. Vaisman [5] defined the second connection closely related to the foliated structure, and showed that there are no nontrivial foliated harmonic 1-forms on $M$ with positive definite Ricci curvature of the second connection. In this note we shall discuss the square-integrable basic harmonic 1 -forms in the complete case and obtain a similar result.

1. Definitions. Let $M$ be an $n$-dimensional $C^{\infty}$-manifold which, topologically, is a connected, orientable, paracompact, Hausdorff space. We shall assume that a foliation $E$ of codimension $q$ is given on $M$, and we may find about each point a coordinate neighbourhood with coordinates $\left(x^{1}, \ldots, x^{p}, y^{1}, \ldots, y^{q}\right)(n=p+q)$ such that

(i) $\left|x^{i}\right| \leqslant 1,\left|y^{\alpha}\right| \leqslant 1$.

(ii) The integral manifolds of $E$ are given locally by $y^{1}=c^{1}, \ldots, y^{q}=c^{q}$ for constants $c^{\alpha}$ satisfying $\left|c^{\alpha}\right| \leqslant 1$. (Here and hereafter, Latin indices run from 1 to $p$ and Greek indices from 1 to $q$.)

Such a coordinate neighbourhood will be called flat, while each of the slices given by a set of equations $y^{\alpha}=c^{\alpha}$ will be called a plaque.

We may assume that there exist in a flat neighbourhood $U$ differential forms $w^{i}$ and vectors $v_{\alpha}$ such that

(i) $\left\{\partial / \partial x^{i}\right\}$ forms the base for the space of cross-sections of $E$ in $U$ at each point.

(ii) $\left\{w^{1}, \ldots, w^{p}, d y^{1}, \ldots, d y^{q}\right\}$ and $\left\{\partial / \partial x^{1}, \ldots, \partial / \partial x^{p}, v_{1}, \ldots, v_{q}\right\}$ are dual bases for the cotangent and tangent spaces at each point of $U$ respectively. Hence, $w^{i}=d x^{i}+\sum a_{\alpha}^{i} d y^{\alpha}$ and $v_{\alpha}=\partial / \partial y^{\alpha}+\sum b_{\alpha}^{i} \partial / \partial x^{i}$.

Received by the editors December 3, 1979.

AMS (MOS) subject classifications (1970). Primary 57D30.

Key words and phrases. Bundle-like metric, second connection, $\square$ "-harmonic form.

(C) 1980 American Mathematical Society 0002-9947/80/0000-0556/\$02.75 
Throughout this note, all local expressions for differential forms and vectors will be taken with respect to those bases.

2. Square-integrable basic cohomology spaces. On a foliated manifold we may have the decomposition of differential forms into components in the following way. Any $C^{\infty}-m$-form $\phi$ may be expressed locally as

$$
\sum_{\substack{i_{1}<\cdots<i_{r} \\ \alpha_{1}<\cdots<\alpha_{s}}} \sum_{r+s=m} \phi_{i_{1} \cdots i_{r} \alpha_{1} \cdots \alpha_{s}}(x, y) w^{i_{1}} \wedge \cdots \wedge w^{i_{r}} \wedge d y^{\alpha_{1}} \wedge \cdots \wedge d y^{\alpha_{s}} .
$$

We may define $\Pi_{r, s} \phi$ to be the sum of all these terms with a fixed $r$ and $s$. Since under a change of flat coordinate systems, $\left\{\left\{d y^{\alpha}\right\}\right\}$ goes into $\left\{\left\{d y^{* \alpha}\right\}\right\}$ and $\left\{\left\{w^{i}\right\}\right\}$ into $\left\{\left\{w^{* i}\right\}\right\}$, the operator $\Pi_{r, s}$ is independent of the choice of coordinate system. Here by $\{\{\cdot\}\}$ we mean the vector space generated by the set $\{\cdot\} . \Pi_{r, s} \phi$ is called the component of type $(r, s)$ of $\phi$. The type decomposition of forms induces a type decomposition of the exterior derivative $d$ by the rule $\left(\Pi_{t, u} d\right) \phi=$ $\sum_{r, s} \Pi_{r+t, s+u} d \Pi_{r, s} \phi$. Letting $\Pi_{1,0} d=d^{\prime}, \Pi_{0,1} d=d^{\prime \prime}$ and $\Pi_{-1,2} d=d^{\prime \prime \prime}$, we have $d=d^{\prime}+d^{\prime \prime}+d^{\prime \prime \prime}$.

Proposition 2.1 (CF. [4]). If $\phi$ is of type $(0, s)$, then $d \phi=d^{\prime} \phi+d^{\prime \prime} \phi$. Moreover, $d^{\prime} \phi=0$ if and only if $\phi$ depends only upon $y$, in the sense that locally

$$
\phi=\sum \phi_{\alpha_{1} \cdots \alpha_{s}}(y) d y^{\alpha_{1}} \wedge \cdots \wedge d y^{\alpha_{s}} .
$$

Definition 2.1. A form of type $(0, s)$ which is annihilated by $d^{\prime}$ will be called a basic form.

Definition 2.2. A riemannian metric (, ) is bundle-like if it is representable in each flat neighbourhood $U$ by an expression of the form

$$
\left.(,)\right|_{U}=\sum g_{i j}(x, y) w^{i} \cdot w^{j}+\sum g_{\alpha \beta}(y) d y^{\alpha} \cdot d y^{\beta} .
$$

Hereafter, we assume that the riemannian metric on $M$ is bundle-like and all leaves are compact.

Let $\Lambda^{0, s}(M)$ be the space of all $C^{\infty}$-basic forms of type $(0, s)$ and $\Lambda_{0}^{0, s}(M)$ the subspace of $\Lambda^{0, s}(M)$ composed of forms with compact support. Restricted to $\Lambda^{0, *}(M)=\sum_{s=0}^{\infty} \Lambda^{0, s}(M), d^{\prime \prime 2}=d^{2}=0$, so we may consider the cohomology of $\Lambda^{0, *}(M)$ and $d^{\prime \prime}$. (This is called the base-like cohomology by B. L. Reinhart [4].)

B. L. Reinhart [4] introduces the *"-operation on $\Lambda^{0, s}(M)$, and defined by

$$
\begin{aligned}
& \text { *" } \phi=\sum_{\substack{\alpha_{1}<\cdots<\alpha_{s} \\
\beta_{1}<\cdots<\beta_{q-s}}} \operatorname{sgn}\left(\begin{array}{ccc}
1 & \ldots \ldots, & q \\
\alpha_{1}, \ldots, \alpha_{s}, \beta_{1}, \ldots, \beta_{q-s}
\end{array}\right)\left(\operatorname{det}\left(g_{\alpha \beta}\right)\right)^{1 / 2} \\
& \cdot g^{\alpha_{1} \nu_{1}} \cdots g^{\alpha_{s} \nu_{s}} \phi_{\nu_{1}} \cdots \nu_{s} d y^{\beta_{1}} \wedge \cdots \wedge d y^{\beta_{q-s}} .
\end{aligned}
$$

According to B. L. Reinhart [4], we may define a riemannian metric on $\Lambda^{0, s}(M)$ by

$$
\langle\phi, \psi\rangle=\phi \wedge^{* \prime} \psi \wedge d x^{1} \wedge \cdots \wedge d x^{p}
$$


and obtain a pre-Hilbertian metric on $\Lambda_{\circ}^{0, s}(M)$ by

$$
\langle\langle\phi, \psi\rangle\rangle=\int_{M}\langle\phi, \psi\rangle=\int_{M} \phi \wedge^{* \prime \prime} \psi \wedge d x^{1} \wedge \cdots \wedge d x^{p} .
$$

The differential operator $d^{\prime \prime} \operatorname{maps} \Lambda^{0, s}(M)$ into $\Lambda^{0, s+1}(M)$. We define $\delta^{\prime \prime}$ : $\Lambda^{0, s}(M) \rightarrow \Lambda^{0, s-1}(M)$ by

$$
\delta^{\prime \prime} \phi=(-1)^{q s+q+1} * \prime d^{\prime \prime * \prime \prime} \phi .
$$

Then we have

$$
\left\langle\left\langle d^{\prime \prime} \phi, \psi\right\rangle\right\rangle=\left\langle\left\langle\phi, \delta^{\prime \prime} \psi\right\rangle\right\rangle
$$

for $\phi \in \Lambda_{\circ}^{0, s}(M), \psi \in \Lambda_{\circ}^{0, s+1}(M)$.

Let $L_{2}^{0, s}(M)$ be the completion of $\Lambda_{\circ}^{0, s}(M)$ with respect to the inner product $\langle\langle\rangle$,$\rangle . We will denote by \partial$ the restriction of $d^{\prime \prime}$ to $\Lambda_{\circ}^{0, s}(M)$ and by $\theta$ the restriction of $\delta^{\prime \prime}$ to $\lambda_{\circ}^{0, s}(M)$. Define $\bar{\partial}=(\theta)^{*}$ and $\bar{\theta}=(\bar{\partial})^{*}$ where ()$^{*}$ denotes the adjoint operator of () with respect to the inner product $\langle\langle\rangle$,$\rangle . Then \bar{\partial}$ (resp. $\bar{\theta}$ ) is a closed, densely defined operator of $L_{2}^{0, s}(M)$ into $L_{2}^{0, s+1}(M)$ (resp. $\left.L_{2}^{0, s-1}(M)\right)$. Let $D_{\bar{\partial}}^{0, s}$ (resp. $\left.D_{\bar{\theta}}^{0, s}\right)$ be the domain of the operator $\bar{\partial}(\operatorname{resp} . \bar{\theta})$ in $L_{2}^{0, s}(M)$. We put

$$
Z_{\bar{\partial}}^{0, s}(M)=\left\{\phi \in D_{\bar{\partial}}^{0, s} \mid \bar{\partial} \phi=0\right\} \quad \text { and } Z_{\bar{\theta}}^{0, s}(M)=\left\{\phi \in D_{\bar{\theta}}^{0, s} \mid \bar{\theta} \phi=0\right\}
$$

which are closed in $L_{2}^{0, s}(M)$. Let $B_{\bar{\partial}}^{0, s}(M)$ and $B_{\bar{\theta}}^{0, s}(M)$ be the closure of $\bar{\partial}\left(D_{\bar{\partial}}^{0, s-1}\right)$ and $\bar{\theta}\left(D_{\bar{\theta}}^{0, s+1}\right)$ respectively.

Definition 2.3. $H_{2}^{0, s}(M)=Z_{\vec{\partial}}^{0, s}(M) \ominus B_{\partial}^{0, s}(M)$ is the square-integrable basic cohomology space, where $\ominus$ denotes the orthogonal complement of $B_{\vec{\partial}}^{0, s}(M)$.

Theorem 2.1 (CF. [1]) (The Orthogonal Decomposition Theorem).

$$
L_{2}^{0, s}(M)=H_{2}^{0, s}(M) \oplus B_{\partial}^{0, s}(M) \oplus B_{\bar{\theta}}^{0, s}(M) .
$$

Definition 2.4. The Laplacian acting on $\Lambda^{0, *}(M)$ is defined by $\square^{\prime \prime}=d^{\prime \prime} \delta^{\prime \prime}+$ $\delta^{\prime \prime} d^{\prime \prime}$.

Proposition 2.2 (CF. [1]). Let the bundle-like metric on $M$ be complete and all leaves be compact. If $\phi \in L_{2}^{0, s}(M) \cap \Lambda^{0, s}(M)$ such that $\square^{\prime \prime} \phi=0$, then $d^{\prime \prime} \phi=0$ and $\delta^{\prime \prime} \phi=0$.

THEOREM 2.2 (CF. [1]). Let the bundle-like metric on $M$ be complete and all leaves be compact. If $\phi \in L_{2}^{0, s}(M) \cap \Lambda^{0, s}(M)$ such that $\square^{\prime \prime} \phi=0$, then $\phi \in H_{2}^{0, s}(M)$.

3. The second connection. According to I. Vaisman (cf. [2], [5]) we define the second connection $D$ on $M$ induced from the bundle-like metric (, ) as follows.

$$
\begin{aligned}
D_{\partial / \partial x^{i}} \partial / \partial x^{j} & =\Gamma_{i j}^{k} \partial / \partial x^{k}, \quad D_{v_{\alpha}} \partial / \partial x^{j}=\Gamma_{\alpha j}^{k} \partial / \partial x^{k}, \\
D_{\partial / \partial x^{i} v_{\beta}} & =0, \quad D_{v_{\alpha}} v_{\beta}=\Gamma_{\alpha \beta}^{\gamma} v_{\gamma}, \\
\left(\partial / \partial x^{i}\right)\left(\partial / \partial x^{j}, \partial / \partial x^{k}\right) & =\left(D_{\partial / \partial x^{i}} \partial / \partial x^{j}, \partial / \partial x^{k}\right)+\left(\partial / \partial x^{j}, D_{\partial / \partial x^{i}} \partial / \partial x^{k}\right),
\end{aligned}
$$




$$
\begin{array}{rlrl}
v_{\alpha}\left(v_{\beta}, v_{\gamma}\right) & =\left(D_{v_{\alpha}} v_{\beta}, v_{\gamma}\right)+\left(v_{\beta}, D_{v_{\alpha}} v_{\gamma}\right), \\
T\left(\partial / \partial x^{i}, \partial / \partial x^{j}\right) & =0, & T\left(\partial / \partial x^{i}, v_{\beta}\right)=0, \\
T\left(v_{\alpha}, \partial / \partial x^{j}\right) & =0, & T\left(v_{\alpha}, v_{\beta}\right)=T_{\alpha \beta}^{k} \partial / \partial x^{k},
\end{array}
$$

where $T$ denotes the torsion tensor of $D$; that is, for any vector fields $X, Y$ on $M$, $T(X, Y)=D_{X} Y-D_{Y} X-[X, Y]$. Note that the torsion $T$ of $D$ does not always vanish. Then we get

$$
\begin{aligned}
\Gamma_{i j}^{k} & =\Gamma_{j i}^{k}=\frac{1}{2} g^{h k}\left(\partial g_{h j} / \partial x^{i}+\partial g_{i h} / \partial x^{j}-\partial g_{i j} / \partial x^{h}\right), \\
\Gamma_{\alpha \beta}^{\gamma} & =\Gamma_{\beta \alpha}^{\gamma}=\frac{1}{2} g^{\tau \gamma}\left(v_{\alpha}\left(g_{\tau \beta}\right)+v_{\beta}\left(g_{\alpha \tau}\right)-v_{\tau}\left(g_{\alpha \beta}\right)\right), \\
\Gamma_{\alpha j}^{k} & =-\partial b_{\alpha}^{k} / \partial x^{j} \\
\partial \Gamma_{\alpha \beta}^{\gamma} / \partial x^{i} & =0 .
\end{aligned}
$$

REMARK 1. If the transversal (or normal) plane field $E^{\perp}$ to $E$ with respect to (, ) is integrable, then the second connection coincides with the Levi-Civita connection induced from ( , ) (cf. [5]).

REMARK 2. $\Gamma_{i j}^{k}$ and $\Gamma_{\alpha \beta}^{\gamma}$ coincide with the coefficients of the Levi-Civita connection (cf. [5]).

We express the operators $d^{\prime \prime}, \delta^{\prime \prime}$ and $\square^{\prime \prime}$ in terms of the second connection $D$. For any $\phi=\Sigma \phi_{\alpha_{1}} \cdots \alpha_{s}(y) d y^{\alpha_{1}} \wedge \cdots \wedge d y^{\alpha_{s}} \in \Lambda^{0, s}(M)$,

$$
\begin{aligned}
\left(d^{\prime \prime} \phi\right)_{\beta_{1}} \cdots \beta_{s+1}= & \sum_{\nu=1}^{s+1}(-1)^{\nu-1} D_{\beta_{v}} \phi_{\beta_{1}} \cdots \hat{\beta}_{\nu} \cdots \beta_{s+1}, \\
\left(\delta^{\prime \prime} \phi\right)_{\beta_{1}} \cdots \beta_{s-1}= & -D^{\gamma} \phi_{\gamma \beta_{1}} \cdots \beta_{s-1}, \\
\left(\square^{\prime \prime} \phi\right)_{\alpha_{1}} \cdots \alpha_{s}= & -D^{\alpha} D_{\alpha} \phi_{\alpha_{1}} \cdots \alpha_{s}+\sum_{k=1}^{s}(-1)^{k} R_{\cdot \alpha \cdot \alpha_{k}}^{\gamma \cdot \alpha \cdot} \phi_{\gamma \alpha_{1}} \cdots \hat{\alpha}_{k} \cdots \alpha_{s} \\
& +2 \sum_{h<k}(-1)^{h+k} R_{\cdot \alpha_{h} \cdot \alpha_{k}}^{\gamma \cdot \alpha \cdot} \phi_{\alpha \gamma \alpha_{1} \cdots \hat{\alpha}_{h} \cdots \hat{\alpha}_{k} \cdots \alpha_{s}},
\end{aligned}
$$

where $R$ denotes the curvature tensor of $D$; that is,

$$
R\left(X_{A}, X_{B}\right) X_{C}=D_{X_{A}} D_{X_{B}} X_{C}-D_{X_{B}} D_{X_{A}} X_{C}-D_{\left[X_{A}, X_{B}\right]} X_{C}=R_{C A B}^{F} X_{F}
$$

for $X_{A}=\partial / \partial x^{i}$ or $v_{\alpha}$.

4. $\square$ "-harmonic 1-forms. A differentiable curve $C:[0,1] \rightarrow M$ is said to be tranversal if $\dot{C}(t)$ is in the transversal plane field $E^{\perp}$ for all $t$, where $\dot{C}(t)$ denotes the differential with respect to the parameter $t$. Let $C$ be a transversal curve in $M$. Then, taking its local expression $C(t)=\left(C^{i}(t), C^{\alpha}(t)\right)$, we have

$$
\begin{aligned}
\dot{C}(t) & =\dot{C}^{i}(t) \partial / \partial x^{i}+\dot{C}^{\alpha}(t) \partial / \partial y^{\alpha} \\
& =\left(\dot{C}^{i}(t)-b_{\alpha}^{i} \dot{C}^{\alpha}(t)\right) \partial / \partial x^{i}+\dot{C}^{\alpha}(t) v_{\alpha} \\
& \left.=\dot{C}^{\alpha}(t) v_{\alpha} \quad \text { (by the transversality of } C(t)\right) .
\end{aligned}
$$


A transversal curve $C$ is called a geodesic if

$$
D_{\dot{C}(t)} \dot{C}(t)=\left(\frac{d^{2} C^{\alpha}(t)}{d t^{2}}+\Gamma_{\beta \gamma}^{\alpha} \frac{d C^{\beta}(t)}{d t} \frac{d C^{\gamma}(t)}{d t}\right) v_{\alpha}=0
$$

By a routine calculation, we may show that a transversal geodesic with respect to the second connection coincides with one with respect to the Levi-Civita connection. Hence a geodesic transversal to a leaf is transversal to all leaves (cf. [3]).

We fix a point $o$ in $M$, and for each point $p$ in $M$, we denote by $\rho(p)$ the distance between leaves through $o$ and $p$.

We consider a differentiable function $\mu$ on $\mathbf{R}$ (the reals) satisfying

(i) $0 \leqslant \mu \leqslant 1$ on $\mathbf{R}$,

(ii) $\mu(t)=1$ for $t \leqslant 1$,

(iii) $\mu(t)=0$ for $t \geqslant 2$.

Then we set

$$
w_{k}(p)=\mu(\rho(p) / k) \text { for } k=1,2,3, \ldots
$$

LEMMA 4.1 (CF. [1]). Under the above notations, there exists a number $A$ depending only on $\mu$, such that

(i) $\left\|d^{\prime \prime} w_{k} \wedge \phi\right\|^{2} \leqslant q A^{2}\|\phi\|^{2} / k^{2}$,

(ii) $\left\|d^{\prime \prime} w_{k} \wedge^{* \prime \prime} \phi\right\|^{2} \leqslant q A^{2}\|\phi\|^{2} / k^{2}$, for all $\phi \in \Lambda_{\circ}^{0, s}(M)$, where $\|\phi\|^{2}=\langle\langle\phi, \phi\rangle\rangle$.

For any $\phi \in L_{2}^{0,1}(M) \cap \Lambda^{0,1}(M)$, we have

$$
\left(d^{\prime \prime} \phi, d^{\prime \prime} \psi\right)_{B(k)}+\left(\delta^{\prime \prime} \phi, \delta^{\prime \prime} \psi\right)_{B(k)}=\left(\square^{\prime \prime} \phi, \psi\right)_{B(k)}
$$

for all $\psi \in \Lambda_{B(k)}^{0,1}(M)$, where $\Lambda_{B(k)}^{0,1}(M)$ is the space of all forms of type $(0,1)$ with compact support in $B(k)$ and $B(k)$ is an open tube of radius $k$ of the leaf through the fixed point $o$ in $M$. For $\psi=w_{k}^{2} \phi$, we have

$$
\begin{aligned}
& d^{\prime \prime} \psi=w_{k}^{2} d^{\prime \prime} \phi+2 w_{k} d^{\prime \prime} w_{k} \wedge \phi, \\
& \delta^{\prime \prime} \psi=w_{k}^{2} \delta^{\prime \prime} \phi-{ }^{* \prime \prime}\left(2 w_{k} d^{\prime \prime} w_{k} \wedge^{* \prime \prime} \phi\right)
\end{aligned}
$$

We consider the 1 -form $\Phi$ of type $(0,1)$ defined by

$$
\Phi=\left(D_{\alpha} \phi_{\beta}\right) \phi^{\beta} d y^{\alpha}=g^{\beta \gamma}\left(D_{\alpha} \phi_{\beta}\right) \phi_{\gamma} d y^{\alpha}
$$

for $\phi \in L_{2}^{0,1}(M) \cap \Lambda^{0,1}(M)$. Since $w_{k}^{2} \Phi$ is compactly supported in $B(2 k)$, the Stokes formula gives the equality

$$
\int_{M}^{* \prime \prime} \delta^{\prime \prime}\left(w_{k}^{2} \Phi\right) \wedge d x^{1} \wedge \cdots \wedge d x^{p}=0
$$

In fact, ${ }^{* \prime \prime}\left(w_{k}^{2} \Phi\right) \wedge d x^{1} \wedge \cdots \wedge d x^{p}$ being a form of type $(p, q-1)$ with compact support in $B(2 k)$,

$$
\int_{M} d\left({ }^{* \prime \prime}\left(w_{k}^{2} \Phi\right) \wedge d x^{1} \wedge \cdots \wedge d x^{p}\right)=0
$$


And

$$
\begin{aligned}
d\left({ }^{* \prime \prime}\left(w_{k}^{2} \Phi\right) \wedge\right. & \left.d x^{1} \wedge \cdots \wedge d x^{p}\right) \\
& =\left(d^{\prime}+d^{\prime \prime}+d^{\prime \prime \prime}\right)\left(* \prime \prime\left(w_{k}^{2} \Phi\right) \wedge d x^{1} \wedge \cdots \wedge d x^{p}\right) \\
& =d^{\prime \prime}\left({ }^{* \prime}\left(w_{k}^{2} \Phi\right) \wedge d x^{1} \wedge \cdots \wedge d x^{p}\right) \\
& ={ }^{* \prime \prime}\left({ }^{* \prime \prime} d^{\prime \prime} *^{\prime \prime}\left(w_{k}^{2} \Phi\right) \wedge d x^{1} \wedge \cdots \wedge d x^{p}\right) \\
& =-^{* \prime \prime} \delta^{\prime \prime}\left(w_{k}^{2} \Phi\right) \wedge d x^{1} \wedge \cdots \wedge d x^{p} .
\end{aligned}
$$

By (3.3.2) and (4.2.2), (4.3) becomes the equality $\left\langle\left\langle 2 w_{k} d^{\prime \prime} w_{k} \wedge \phi, D \phi\right\rangle\right\rangle_{B(2 k)}+\left\langle\left\langle w_{k} D^{2} \phi, w_{k} \phi\right\rangle\right\rangle_{B(2 k)}+\left\langle\left\langle w_{k} D \phi, w_{k} D \phi\right\rangle\right\rangle_{B(2 k)}=0$, where $\left(D^{2} \phi\right)_{\beta}=D^{\alpha} D_{\alpha} \phi_{\beta}$. And (3.3.3) gives the equality

$$
\left\langle\left\langle\square^{\prime \prime} \phi, w_{k}^{2} \phi\right\rangle\right\rangle_{B(2 k)}=-\left\langle\left\langle w_{k} D^{2} \phi, w_{k} \phi\right\rangle\right\rangle_{B(2 k)}+\left\langle\left\langle w_{k} \Re \phi, w_{k} \phi\right\rangle\right\rangle_{B(2 k)},
$$

where $R$ is the symmetric linear transformation on 1 -forms defined by $(\Re \phi)_{\beta}=$ $-R_{\cdot \boldsymbol{\alpha} \cdot \boldsymbol{\beta}}^{\gamma \cdot \boldsymbol{\alpha}} \phi_{\gamma}$.

On the other hand, the Schwartz inequality and Lemma 4.1 give the following.

$$
\begin{aligned}
\left|\left\langle\left\langle 2 w_{k} d^{\prime \prime} w_{k} \wedge \phi, D \phi\right\rangle\right\rangle_{B(2 k)}\right| & \leqslant \frac{2 q^{1 / 2} A}{k}\left\|w_{k} D \phi\right\|_{B(2 k)}\|\phi\|_{B(2 k)} \\
& \leqslant \frac{q^{1 / 2} A}{k}\left(\left\|w_{k} D \phi\right\|_{B(2 k)}^{2}+\|\phi\|_{B(2 k)}^{2}\right),
\end{aligned}
$$

and

$$
\left|\left\langle\left\langle\square^{\prime \prime} \phi, w_{k}^{2} \phi\right\rangle\right\rangle_{B(2 k)}\right| \leqslant \frac{1}{2}\left(\frac{1}{\sigma}\left\|w_{k} \phi\right\|_{B(2 k)}^{2}+\sigma\left\|w_{k} \square^{\prime \prime} \phi\right\|_{B(2 k)}^{2}\right)
$$

for every $\sigma>0$.

Then we have

$$
\begin{aligned}
\sigma\left\|w_{k} \square^{\prime \prime} \phi\right\|_{B(2 k)}^{2}+\frac{1}{\sigma}\left\|w_{k} \phi\right\|_{B(2 k)}^{2} \geqslant & 2\left\langle\left\langle w_{k} \Re \phi, w_{k} \phi\right\rangle\right\rangle_{B(2 k)}-\frac{2 q^{1 / 2} A}{k}\|\phi\|_{B(2 k)}^{2} \\
& +2\left(1-q^{1 / 2} A / k\right)\left\|w_{k} D \phi\right\|_{B(2 k)}^{2}
\end{aligned}
$$

In particular, setting $\square^{\prime \prime} \phi=0$ and letting $\sigma \rightarrow \infty$, we have

$$
\begin{aligned}
0 \geqslant & 2\left\langle\left\langle w_{k} \Re \phi, w_{k} \phi\right\rangle\right\rangle_{B(2 k)}-2 q^{1 / 2} A / k\|\phi\|_{B(2 k)}^{2} \\
& +2\left(1-q^{1 / 2} A / k\right)\left\|w_{k} D \phi\right\|_{B(2 k)}^{2} .
\end{aligned}
$$

Letting $k \rightarrow \infty$, we have

$$
0 \geqslant \limsup _{k \rightarrow \infty}\left\langle\left\langle w_{k} \Re \phi, w_{k} \phi\right\rangle\right\rangle_{B(2 k)}+\|D \phi\|^{2} .
$$

Suppose that the minimal eigenvalue $\lambda$ of $\mathscr{R}$ is nonnegative. Then there exists a constant $K \geqslant 0$ satisfying

$$
\limsup _{k \rightarrow \infty}\left\langle\left\langle w_{k} \Re \phi, w_{k} \phi\right\rangle\right\rangle_{B(2 k)} \geqslant K\|\phi\|^{2} .
$$

In fact, there exists a constant $K \geqslant 0$ such that $\lambda \geqslant K$. Hence we have $0>\|D \phi\|^{2}$ $+K\|\phi\|^{2}$. 
Definition 4.1. A basic form $\phi$ of type $(0, s)$ is $\square$ "-harmonic if $\square$ " $\phi=0$.

Definition 4.2. A basic l-form $\phi$ of type $(0,1)$ is $D$-parallel if $D \phi=0$.

Therefore we have

MAIN THEOREM. Let the bundle-like metric on $M$ be complete and all leaves be compact. If the minimal eigenvalue $\lambda$ of the Ricci tensor $-R_{\cdot \alpha \cdot \beta}^{\gamma \cdot \alpha \cdot}$ is positive and bounded away from zero, then there are no nontrivial basic $\square^{\prime \prime}$-harmonic 1-forms in $L_{2}^{0,1}(M)$. Moreover, if $\lambda$ is zero, then the basic $\square^{\prime \prime}$-harmonic 1 -form of type $(0,1)$ is D-parallel.

REMARK. "There are no nontrivial basic $\square$ "-harmonic 1-forms in $L_{2}^{0,1}(M)$ " means that we consider the operator $\square^{\prime \prime}$ in $L_{2}^{0,1}(M)$ only and we do not consider the extension $\bar{\square}^{\prime \prime}$ to $L_{2}^{0,1}(M)$.

If we consider the operator $\square^{\prime \prime}$ in $\Lambda^{0,1}(M)$, we may have the following statement. Let the bundle-like metric on $M$ be complete. If the minimal eigenvalue $\lambda$ of the Ricci tensor $-R_{\cdot \alpha \cdot \beta}^{\gamma \cdot \alpha \cdot}$ is positive and bounded away from zero, then there are no nontrivial global square-integrable basic $\square^{\prime \prime}$-harmonic forms $\phi$ of type $(0,1)$ such that $\left\|d^{\prime \prime} \phi\right\|<\infty$ and $\left\|\delta^{\prime \prime} \phi\right\|<\infty$.

\section{REFERENCES}

1. H. Kitahara, Remarks on square-integrable basic cohomology spaces on a foliated riemannian manifold, Kōdai Math. J. 2 (1979), 187-193.

2. On a parametrix form in a certain V-submersion, (Proc. Conf. Geometry, Haifa), Lecture Notes in Math., vol. 792, Springer-Verlag, Berlin and New York, 1980.

3. B. L. Reinhart, Foliated manifolds with bundle-like metrics, Ann. of Math. (2) 69 (1959), 119-132.

4. __ Harmonic integrals on foliated manifolds, Amer. J. Math. 81 (1959), 529-536.

5. I. Vaisman, Variétés riemanniennes feuilletées, Czechoslovak Math. J. 21 (1971), 46-75.

Department of Mathematics, College of Liberal Arts, Kanazawa University, Kanazawa 920 , JAPAN 